\title{
Optimal Trajectory Tracking Control for Underwater Profiling Monitoring Vehicle
}

\author{
J. Yuan \\ National Engineering and Technological Research Center of \\ Marine Monitoring Equipment \\ Qingdao Shandong Provincial Key Laboratory of Ocean \\ Environment Monitoring Technology \\ Qingdao, China \\ Institute of Oceanographic Instrumentation of Shandong \\ Academy of Sciences \\ Qingdao, China
}

\author{
C. Hui \\ National Engineering and Technological Research Center of \\ Marine Monitoring Equipment \\ Qingdao, China \\ Shandong Provincial Key Laboratory of Ocean Environment \\ Monitoring Technology \\ Qingdao, China \\ Institute of Oceanographic Instrumentation of Shandong \\ Academy of Sciences \\ Qingdao, China
}

\begin{abstract}
The optimal trajectory-tracking control method for the underwater profiling monitoring vehicle (UPMV) is investigated. Firstly, the system dynamics of the UPMV is modelled and the quadratic performance index is chosen for considering the tracking control precision and energy consumption. The optimal tracking control law is proposed based on Hamilton function. Simulation results show the effectiveness of the control scheme.
\end{abstract}

Keywords-underwater profiling monitoring vehicle; trajectorytracking; dynamics; Hamilton function

\section{INTRODUCTION}

Marine environmental monitoring technology are of great significance in the development of marine resources, marine disaster protection, protection of the marine environment $[1,2]$. Marine monitoring technology has entered into the integrated three-dimensional monitoring way. Aviation and aerospace remote sensing technology have solved certain extents from the air to the sea surface in real-time synchronization and large-scale observation. The sea observation stations, offshore mooring buoy and bottom-seabed based observing systems solve the fixed-point observation; the surveying vessels and drifting buoys, although used in the observation on section observation, can not be used in a specific area, such as the danger zone and the sensitive region in ref. $[3,4,5]$. The marine environment section observing in our country still exist certain technical limitations, such as the weaker sampling capability of underwater monitoring equipment, low frequency of monitoring and lacking of mobile monitoring capabilities. Secondly, the observing area is limited to the monitoring of the sea surface, lacking of a certain dynamic monitoring means or it is limited to certain fixed vertical profile monitoring waters, lacking of a wide range of mobile monitoring capability. Underwater profiling observation vehicle becomes one of the means to fill the observation gaps. Underwater profiling monitoring vehicle can achieve a tracking observation on predetermined profiling trajectory, the trajectory tracking control technology is the key technology to achieve high-precision profiling observation, but the underwater vehicle itself has limited battery energy. In this paper, considering the waypoint tracking precision and driving energy consumption, we propose an optimal tracking control method based on system dynamics model. The simulation results verify the validity of the control method finally.

\section{PROBlem Descriptions}

Firstly, we establish two coordinate frames: the geocentric inertial coordinate frame $E-\xi \eta \zeta$ and the body-fixed coordinate frame $O-x y z$. The origin $E$ in inertial coordinate frame is defined in some point at sea level, the $E-\xi$ and $E-\eta$ are in horizontal level, and $E-\xi$ is parallel with the longitude and points northwardly, $E-\eta$ is parallel with the latitude and points eastwardly, and $E-\zeta$ is perpendicular to horizontal level and points to the earth's core. The three axes form the right-hand screw relation. The origin $O$ in body-fixed coordinate frame is defined in some point at the UPMV cancroids. $O-X$ is defined in longitudinal section plane and points to the orientation of linear velocity, $O-y$ is perpendicular to the portrait section plane and points to the right-hand orientation, and $\mathrm{O}-\mathrm{Z}$ is in the longitudinal section plane and points to the down orientation. Also the three axes form the right-hand screw relation. $\mathrm{O}-\mathrm{x}, \mathrm{O}-\mathrm{y}$ and $O-z$ are inertia principal axis of an UPMV. $x_{p}=\left[\begin{array}{lll}\xi & \eta & \zeta\end{array}\right]^{T}$ denotes the position of UPMV in the inertial coordinate frame, $x_{a}=\left[\begin{array}{lll}\phi & \theta & \varphi\end{array}\right]^{T}$ denotes the attitude of UPMV in the inertial coordinate frame, where $\theta$ denotes the yaw angel, $\phi$ is the pitch angle of UPMV and $\varphi$ is the roll angle of UPMV; $r_{f}=\left[\begin{array}{lll}x_{f} & y_{f} & z_{f}\end{array}\right]^{T}$ denotes the position vector of UPMV floating centre in the fixed-body coordinates, $v=\left[\begin{array}{lll}u & v & w\end{array}\right]^{T}$ denotes the velocity vector in the fixed-body frame, $\dot{v}=\left[\begin{array}{lll}\dot{u} & \dot{v} & \dot{w}\end{array}\right]^{T}$ denotes the acceleration vector in fixed-body frame, 
$\Omega=\left[\begin{array}{lll}p & q & r\end{array}\right]^{T}$ denotes the angular velocity vector and $\dot{\Omega}=\left[\begin{array}{lll}\dot{p} & \dot{q} & \dot{r}\end{array}\right]^{T}$ denotes the angular acceleration vector.

The dynamic equations of the UPMV can be described as follows

$$
\begin{gathered}
M(h) \ddot{h}+V(h, \dot{h}) \dot{h}=u \\
u=A F
\end{gathered}
$$

where $M(h) \in R^{6 \times 6}$ is a symmetric positive definite inertia matrix, $V(h, \dot{h}) \in R^{6 \times 6}$ is the matrix of centrifugal and Coriolis forces, $u \in R^{6}$ is the control input, $A$ is the input matrix and $\boldsymbol{h}=\left[\begin{array}{llllll}\xi & \eta & \zeta & \phi & \theta & \varphi\end{array}\right]^{T}$.

Set $N(h, \dot{h})=V(h, \dot{h}) \dot{h}$, then equation (1) can be written as

$$
M(h) \ddot{h}+N(h, \dot{h})=u,
$$

Introducing a state variable as

$$
x=\left[\begin{array}{l}
x_{1} \\
x_{2}
\end{array}\right]=\left[\begin{array}{l}
h \\
\dot{h}
\end{array}\right]
$$

Then equation (1) can be written as the following state equation form

$$
\dot{x}=f(x)+g(x) u
$$

where

$$
f(x)=\left[\begin{array}{c}
x_{2} \\
-M^{-1}\left(x_{1}\right) N(x)
\end{array}\right], g(x)=\left[\begin{array}{c}
0 \\
-M^{-1}\left(x_{1}\right)
\end{array}\right] .
$$

The predefined trajectory of the UPMV is set as $h_{d}$, the predefined state is $x_{d}=\left[\begin{array}{ll}h_{d} & \dot{h}_{d}\end{array}\right]^{T}$, and the state error is $e=x_{d}-x$, so the error equation is

$$
\dot{e}=\dot{x}_{d}-\dot{x}=\dot{x}_{d}-f(x)-g(x) u .
$$

The performance index is chosen as

$$
J=\frac{1}{2}\left\{e^{T}\left(t_{f}\right) P\left(t_{f}\right) e\left(t_{f}\right)+\int_{t_{0}}^{t_{f}}\left[e^{T}(t) Q(t) e(t)+\dot{e}^{T}(t) R(t) \dot{e}(t) d t\right\}\right.
$$

where $Q(t)$ is semi-definite matrix, $R(t)=\left[\begin{array}{cc}0 & 0 \\ 0 & R_{0}(t)\end{array}\right], R_{0}(t)$ and $P(t)$ is $n \times n$ and $2 n \times 2 n$ positive definite matrix, respectively. The first term in the performance reflects the requirement on the end error, the first integral term reflects the requirement on the control process error and the second reflects the energy consumption requirement

\section{Optimal Control LaW Designing}

For equation (2), we chosen a Hamilton function

$$
\begin{aligned}
& \bar{H}=-\left(\frac{\partial J}{\partial e(t)}\right)^{T} \dot{e}(t)-\frac{1}{2} e^{T}(t) Q(t) e^{T}(t)-\frac{1}{2} \dot{e}^{T}(t) R(t) \dot{e}^{T}(t) \\
& \text { so } \\
& \frac{\partial \bar{H}}{\partial u}=\frac{\partial \dot{e}(t)}{\partial u} \frac{\partial \bar{H}}{\partial \dot{e}(t)}=-g(x)^{T}\left[-\frac{\partial J}{\partial e(t)}-R(t) \dot{e}(t)\right] \\
& \left.=g(x)^{T}\left[\frac{\partial J}{\partial e(t)}+R(t)\right)\left(\dot{x}_{d}-f(x)-g(x) u\right)\right] \\
& =g(x)^{T} \frac{\partial J}{\partial e(t)}+\left[\begin{array}{ll}
0 & \left.H^{-1}\right]
\end{array}\left[\begin{array}{cc}
0 & 0 \\
0 & R_{0}
\end{array}\right]\left[\begin{array}{l}
\dot{h}_{d} \\
\dot{h}_{d}
\end{array}\right]-\left[\begin{array}{c}
\dot{h} \\
-H^{-1} N
\end{array}\right]\right\}-\left[\begin{array}{ll}
0 & H^{-1}
\end{array}\right]\left[\begin{array}{cc}
0 & 0 \\
0 & R_{0}
\end{array}\right]\left[\begin{array}{c}
0 \\
H^{-1}
\end{array}\right] u \\
& =g(x)^{T} \frac{\partial J}{\partial e(t)}+H^{-1} R_{0}\left(\ddot{h_{d}}+H^{-1} N\right)-H^{-1} R_{0} H^{-1} u \\
& \text { Set } \frac{\partial \bar{H}}{\partial u}=0, \text { so we can solve the equation }
\end{aligned}
$$

$$
u=H R_{0}^{-1} H\left[g(x)^{T} \frac{\partial J}{\partial e(t)}+H^{-1} R_{0}\left(\dot{h}_{d}+H^{-1} N\right)=\left[\begin{array}{ll}
0 & H R_{0}^{-1}
\end{array}\right] \frac{\partial J}{\partial e(t)}+H \ddot{h_{d}}+N=u^{*}\right.
$$

because $\frac{\partial \bar{H}}{\partial u}=0$ has a only solution $u^{*}$, and

$\frac{\partial}{\partial u}\left[\left(\frac{\partial \bar{H}}{\partial u}\right)^{T}\right]=\frac{\partial}{\partial u}\left[\left(\frac{\partial \bar{H}}{\partial e(t)}\right)^{T} g(x)+\left(\dot{x}_{d}-f(x)\right)^{T} R(t) g(x)-u^{T} g(x)^{T} R(t) g(x)\right]$

$=-g(x)^{T} R(t) g(x)=-H^{-1} R_{0} H^{-1}<0$

So $u^{*}$ is the optimal control law, we obtain the optimal error equation and optimal Hamilton function $H^{*}$ which are respectively.

$$
\begin{aligned}
\dot{e} & =\dot{x}_{d}-f(x)-g(x) u^{*} \\
= & {\left[\begin{array}{l}
\dot{h_{d}} \\
\ddot{h_{d}}
\end{array}\right]-\left[\begin{array}{c}
\dot{h} \\
-H^{-1} N
\end{array}\right]-\left[\begin{array}{c}
0 \\
H^{-1}
\end{array}\right]\left\{\left[\begin{array}{ll}
0 & H R_{0}^{-1}
\end{array}\right] \frac{\partial J}{\partial e(t)}+H \ddot{h}_{d}+N\right\} } \\
= & {\left[\begin{array}{ll}
0 & I \\
0 & 0
\end{array}\right]\left[\begin{array}{c}
h_{d}-h \\
\dot{h_{d}}-\dot{h}
\end{array}\right]-\left[\begin{array}{cc}
0 & 0 \\
0 & R_{0}^{-1}
\end{array}\right] \frac{\partial J}{\partial e(t)} } \\
= & F e(t)-T \frac{\partial J}{\partial e(t)} \\
H^{*} & =-\left(\frac{\partial J}{\partial e(t)}\right)^{T} \dot{e}-\frac{1}{2} e^{T} Q e-\frac{1}{2} \dot{e}^{T} R \dot{e} \\
= & -\left(\frac{\partial J}{\partial e(t)}\right)^{T} F e+\left(\frac{\partial J}{\partial e(t)}\right)^{T} T \frac{\partial J}{\partial e(t)}-\frac{1}{2} e^{T} Q e-\frac{1}{2}\left(F e-T \frac{\partial J}{\partial e(t)}\right)^{T} R\left(F e-T \frac{\partial J}{\partial e(t)}\right) \\
= & -\frac{1}{2}\left[\frac{\partial J}{\partial e(t)} F e+e^{T} F^{T} \frac{\partial J}{\partial e(t)}-\left(\frac{\partial J}{\partial e(t)}\right)^{T} T \frac{\partial J}{\partial e(t)}+e^{T} Q e\right]
\end{aligned}
$$

So it is necessary for us to solve the $\frac{\partial J}{\partial e(t)}$ in order to solve the optimal control $u^{*}$.

Because of the boundary condition of the Hamilton-Jacobi, we know 


$$
J\left(e\left(t_{f}\right), t_{f}\right)=\frac{1}{2} e\left(t_{f}\right)^{T} P\left(t_{f}\right) e\left(t_{f}\right)
$$

So we set $J\left(e\left(t_{f}\right), t_{f}\right)$ as quadratic index, that is $J(e(t), t)=\frac{1}{2} e(t)^{T} K(t) e(t)$, and

$$
\frac{\partial J(e(t), t)}{\partial t}=\frac{1}{2} e(t)^{T} \dot{K}(t) e(t), \frac{\partial J(e(t), t)}{\partial e(t)}=K(t) e(t)
$$

And submitting the above equation into the HamiltonJacobi equation, we obtain

$\frac{1}{2} e(t)^{T} \dot{K}(t) e(t)+\frac{1}{2}\left[e(t)^{T} K(t) F e(t)+e(t)^{T} F^{T} K(t) e(t)-e(t)^{T} K(t) T K(t) e(t)+e(t)^{T} Q e(t)\right]$

$=\frac{1}{2} e(t)^{T}\left[\dot{K}(t)+K(t) F+F^{T} K(t)-Q+K(t) T K(t)\right] e(t)=0$

o $K(t)$ satisfies the Riccati equation

$$
\dot{K}(t)=-K(t) F-F^{T} K(t)+Q-K(t) T K(t) \quad \text { and }
$$

boundary condition $K\left(t_{f}\right)=P\left(t_{f}\right)$.

So the optimal control is

$$
\begin{aligned}
& u^{*}=\left[\begin{array}{ll}
0 & H R_{0}^{-1}
\end{array}\right] K(t) e(t)+H \ddot{h}_{d}+N \\
& =H\left[\ddot{h}_{d}+R_{0}^{-1} k_{3}\left(\dot{h}_{d}-\dot{h}\right)+R_{0}^{-1} k_{2}\left(h_{d}-h\right)\right]+V(h, \dot{h}) \dot{h}
\end{aligned}
$$

where

$$
\left.K=\left[\begin{array}{cc}
k_{1} & k_{2} \\
k_{2}^{T} & k_{4}
\end{array}\right], \quad\left(k_{1}, k_{2}, k_{3} \text { all are } n \text { bands phalanx }\right)\right)
$$

is a symmetric positive definite matrix.

when $t_{f}=\infty$, we choose $P=0, Q, R$ are constant matrix, then the optimal control law is

$$
u^{*}=H\left[\ddot{h}_{d}+R_{0}^{-1} \tilde{k}_{3}\left(\dot{h}_{d}-\dot{h}\right)+R_{0}^{-1} \tilde{k}_{2}\left(h_{d}-h\right)\right]+V(h, \dot{h}) \dot{h}
$$

where

$$
\left.\tilde{K}=\left[\begin{array}{cc}
\tilde{k}_{1} & \tilde{k}_{2} \\
\tilde{k}_{2}^{T} & \tilde{k}_{4}
\end{array}\right], \quad\left(\tilde{k}_{1}, \tilde{k}_{2}, \tilde{k}_{3} \text { all are } n \text { bands phalanx }\right)\right)
$$

is a symmetric positive definite matrix

$$
\begin{gathered}
\text { and it satisfies the Riccati equation } \\
\tilde{K} F+F^{T} \tilde{K}-Q+\tilde{K} T \tilde{K}=0
\end{gathered}
$$

\section{SiMULATIONS}

The UPMV is set to certain depth with $d=18 \mathrm{~m}$.Simulation results show the effectiveness of the control scheme.
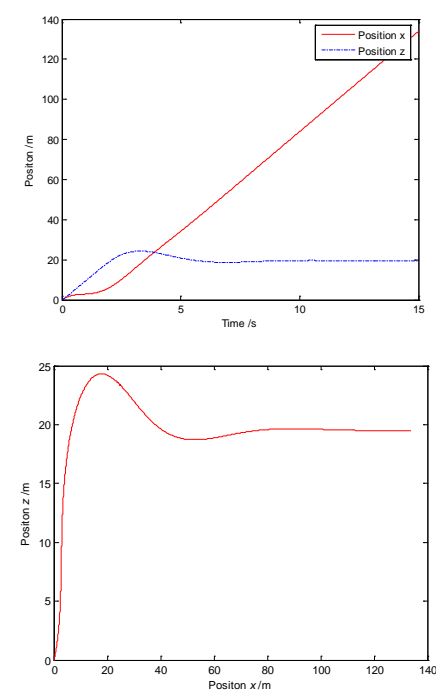

FIGURE I. FIXED-DEPTH CONTROL

\section{CONCLUSIONS}

The trajectory tracking control of the underwater profiling monitoring is a key technology to achieve high-precision profiling observation, but the underwater vehicle has limited battery power. By constructing a system dynamics model and considering the waypoint tracking precision and driving energy consumption, we design an optimal tracking control method which can achieve a predetermined trajectory tracking control precision.

\section{ACKNOWLEDGEMENT}

The work is supported partly by the Shandong Natural Science Foundation (ZR2012FL18), Open Project of Shandong Provincial Key Lab of Marine Ecological Environment and Disaster Prevention and Mitigation (201308), Qingdao Science \& Technology Project (13-1-4-172-jch), Shandong Academy of Sciences Doctoral Foundation (201244) and International Science \& Technology Cooperation Project (2011DFR60810).

\section{REFERENCE}

[1] Wood S, Nulph A, Howell B. Application of autonomous underwater vehicles. Sea Technology, ,45(12), pp.10-14,2004

[2] Ageev M D. Application of solar and wave energies for long-range autonomous underwater vehicles. Advanced Robotics, 16(1), pp. 43-55, 2002

[3] Hemond H F, Mueller A V, Hemond M. Field Testing of Lake Water Chemistry with a Portable and an AUV-based Mass Spectrometer. Journal of the American Society for Mass Spectrometry, 19(10), pp. 1403-1410, 2008

[4] Shome S N, et al. AUV for shallow water applications: Some design aspects. Proceedings of the $18^{\text {th }}$ International Offshore and Polar Engineering Conference, pp. 429-435, 2008

[5] Spiewak J M, Jouvencel B, Fraisse P. A new design of AUV for shallow water applications: H160. Proceedings of The Sixteenth International Offshore and Polar Engineering Conference, pp. 233-238, 2006 